Service social

\title{
Un projet-pilote de soutien aux familles : aux confins de divers courants de recherche
}

\section{Lise Darveau-Fournier et Alice Home}

Volume 35, numéro 1-2, 1986

Recherche - Action - Évaluation

URI : https://id.erudit.org/iderudit/706298ar

DOI : https://doi.org/10.7202/706298ar

Aller au sommaire du numéro

Éditeur(s)

École de service social de l'Université Laval

ISSN

1708-1734 (numérique)

Découvrir la revue

Citer cet article

Darveau-Fournier, L. \& Home, A. (1986). Un projet-pilote de soutien aux

familles : aux confins de divers courants de recherche. Service social, 35(1-2),

126-140. https://doi.org/10.7202/706298ar d'utilisation que vous pouvez consulter en ligne.

https://apropos.erudit.org/fr/usagers/politique-dutilisation/ 
DARVEAU-Fournier, Lise, professeure à l'École de service social de l'Université Laval.

Home, Alice, professeure à l'École de service social de l'Université de Montréal.

\section{Un projet-pilote de soutien aux familles : aux confins de divers courants de recherche}

\section{Lise Darveau-Fournier Alice Home}

La complexité des problèmes sociaux actuels exige souvent l'utilisation d'une combinaison de différents modèles d'intervention afin d'en maximiser les effets. De même, le développement de projets adaptés aux besoins spécifiques d'un milieu gagne parfois à s'appuyer sur plusieurs types de recherche. La collaboration à la conception et à la réalisation d'une intervention de soutien aux familles d'un milieu à risque (Darveau-Fournier, Home et Bédard, 1986) a amené une équipe de travail à identifier certains courants de recherche susceptibles de contribuer à une orientation appropriée du projet. Ceux qui ont été retenus ont trait à la recherche développementale (developmental research and utilization), à la recherche évaluative et à la recherche-action. Cet article vise à présenter les éléments principaux de ces courants et à en illustrer l'application par des exemples concrets.

Notre but n'est donc pas de procéder à une analyse comparative ni de présenter une étude élaborée des publications qui traitent de ces divers modèles de recherche, mais plutôt de nous attarder aux écrits de différents auteurs qui ont apporté des éléments pertinents à l'expérience qui nous concerne.

Nous décrirons d'abord la nature, les objectifs et la structure du projet. Nous présenterons ensuite les principes des trois courants de recherche mentionnés et nous dégagerons leur influence respective. Quelques résultats préliminaires précéderont les conclusions, dans lesquelles certaines difficultés seront soulignées. 


\section{Nature, objectifs et structure}

Le projet-pilote auquel nous référons, et dont vous trouverez un sommaire au Tableau 1, en est un de support aux familles d'un milieu à risque dans lequel se retrouvent un pourcentage élevé de familles monoparentales, une forte proportion de locataires et de nombreux bénéficiaires de prestations gouvernementales (Bellamy et Shookner, 1980). Il a été initié par des intervenantes sociales du Centre local de services communautaires Basse-Ville de Québec et des femmes du milieu concerné (ces dernières étant regroupées en un collectif), auxquelles s'est jointe une équipe de chercheurs en service social.

Le projet s'adresse aux familles qui ont des enfants de 0 à 12 ans et qui rencontrent des problèmes de pauvreté, d'isolement, de santé, de relations parents-enfants. Plusieurs études établissent un lien entre un statut socio-économique faible et un risque élevé de développer des problèmes de santé physique et mentale, de délinquance, de placement d'enfants (Garbarino et Sherman, 1980; Gottlieb, 1981), de même qu'entre le support social et la prévention de la détérioration familiale (Jenkins et Sauber, 1966). Un des objectifs consiste à soulager temporairement les parents dans l'accomplissement de leur rôle. $\AA$ cette fin, un service de gardiennage à court terme (2-15 jours) leur est offert par des familles vivant dans le même quartier (famillesgardiennes). Ce dernier aspect rejoint un autre objectif qui est de maintenir l'enfant dans son milieu de vie, lui permettant ainsi de poursuivre ses activités habituelles lorsqu'il y a lieu (contacts avec ses ami(e)s, école, sports, etc.).

Le projet vise également à dépasser le simple service et à encourager le développement de nouvelles formes de support social entre les familles. Celles qui utilisent le service de gardiennage (familles-utilisatrices) sont invitées à des rencontres de groupe dans le but de se connaître entre elles, de diminuer leur isolement et de se donner de l'information. Des rencontres réunissent mensuellement aussi les familles-gardiennes dans le but de faciliter l'accomplissement de leur tâche, en même temps que d'augmenter leur compétence et leur satisfaction. Familles-utilisatrices et familles-gardiennes sont aussi invitées à se rencontrer ensemble, à certaines occasions.

Enfin, l'expérience vise à s'associer les organismes et groupes communautaires dans la recherche de solutions novatrices aux besoins de soutien des familles. De l'information a été fournie à plusieurs groupes et organismes du milieu ; ils seront bientôt invités à en discuter de façon formelle avec les responsables du projet. 


\section{TABLEAU 1}

\section{Sommaire du projet}

\begin{tabular}{|c|c|c|c|}
\hline Cibles visées & Besoins & Services offerts & Evaluation \\
\hline \multirow[t]{2}{*}{$\begin{array}{l}\text { Parents de jeunes enfants de } \\
\text { la Basse-ville de Québec }\end{array}$} & $\begin{array}{l}\text {-support dans les situations } \\
\text { difficiles }\end{array}$ & $\begin{array}{l}\text {-service de gardiennage de } \\
\text { courte durée (2-15 jours) }\end{array}$ & $\begin{array}{l}\text {-vérifier jusqu'à quel point le } \\
\text { service est utile en période } \\
\text { de transition ou d'incapacité } \\
\text { temporaire }\end{array}$ \\
\hline & -diminution de l'isolement & $\begin{array}{l}\text {-rencontres de groupe pour } \\
\text { partage, support et informa- } \\
\text { tion }\end{array}$ & $\begin{array}{l}\text {-vérifier jusqu'à quel point les } \\
\text { rencontres permettent par- } \\
\text { tage, support et information }\end{array}$ \\
\hline Enfants de 0 à 12 ans & $\begin{array}{l}\text {-maintien dans leur milieu } \\
\text { de vie habituel }\end{array}$ & $\begin{array}{l}\text {-gardiennage dans le quartier } \\
\text { - poursuite des activités habi- } \\
\text { tuelles (école, ami(e)s, voisi- } \\
\text { nage) }\end{array}$ & $\begin{array}{l}\text {-vérifier leurs réactions au } \\
\text { gardiennage et jusqu'à quel } \\
\text { point ils ont maintenu leurs } \\
\text { activités habituelles }\end{array}$ \\
\hline Familles-gardiennes & $\begin{array}{l}\text {-support et formation dans } \\
\text { l'accomplissement de leur } \\
\text { rôle }\end{array}$ & $\begin{array}{l}\text {-rencontres de groupes pour } \\
\text { support et formation }\end{array}$ & $\begin{array}{l}\text {-vérifier jusqu'à quel point les } \\
\text { rencontres de groupe per- } \\
\text { mettent support et formation }\end{array}$ \\
\hline Communauté & $\begin{array}{l}\text {-renforcement des réseaux ou } \\
\text { création de nouveaux réseaux } \\
\text { sociaux } \\
\text {-développement de la capa- } \\
\text { cité du milieu à répondre } \\
\text { aux besoins des familles }\end{array}$ & $\begin{array}{l}\text {-contacts entre familles-gar- } \\
\text { diennes et familles-utilisatrices } \\
\text {-organismes et associations } \\
\text { dans: } \\
\text { - étude des besoins des fa- } \\
\text { milles } \\
\text { - recherche des moyens ap- } \\
\text { propriés pour y répondre }\end{array}$ & $\begin{array}{l}\text {-vérifier l'impact des services } \\
\text { offerts : } \\
\text { - sur les réseaux sociaux des } \\
\text { familles du milieu } \\
\text { - sur les ressources commu- } \\
\text { nautaires et leur engage- } \\
\text { ment dans le support aux } \\
\text { familles }\end{array}$ \\
\hline
\end{tabular}


Comme dans toute expérience-pilote, l'évaluation constitue un volet important. Le tableau 2 présente le devis d'évaluation qui nous concerne.

Comme on peut le voir, l'évaluation porte d'abord sur la pertinence des objectifs du projet afin d'en vérifier la conformité avec les besoins exprimés par les familles du milieu. Elle examinera aussi le degré de réalisation du programme, tant dans son volet "service de gardiennage" que dans celui des "rencontres de groupes", et considérera les possibilités d'en répéter l'application dans d'autres milieux. Enfin, elle tentera d'identifier les effets ou les résultats du programme sur les diverses cibles visées: parents, enfants, famillesgardiennes, milieu. L'évaluation devrait permettre, en particulier, de mesurer l'impact du service de gardiennage sur les familles-utilisatrices, sur la poursuite des relations et des activités quotidiennes de l'enfant, de même que sur le développement de réseaux sociaux entre les familles-gardiennes et les familles-utilisatrices. Elle devrait également permettre de vérifier dans quelle mesure les rencontres de groupe contribuent à briser l'isolement des familles et à leur apporter soutien, information et formation. Pour terminer, l'évaluation vérifiera le degré de connaissance du projet qu'ont les organismes et associations du milieu, et surtout les formes de soutien aux familles qu'ils préconisent.

Mentionnons enfin que les instruments de cueillette de données que nous utilisons sont variés et contiennent à la fois des éléments de types qualitatif et quantitatif. L'évaluation se poursuit selon un processus continu, de façon à permettre l'utilisation des résultats pour ajuster, au fur et à mesure, les objectifs, les moyens et le déroulement de cette intervention.

\section{Appuis de la recherche}

Le projet que nous venons de décrire vise à la fois la mise sur pied d'un nouveau service, le développement de la communauté dans laquelle il s'insère, et l'évaluation de son implantation ainsi que de certains de ses résultats. Dans la section qui suit, nous expliquons pourquoi et comment nous nous sommes appuyées sur des principes issus de trois courants: la recherche développementale, la recherche évaluative et la recherche-action. Puisque le projet, dans sa forme actuelle, a débuté en 1984, nous avons utilisé certains ouvrages disponibles à cette époque. Nous ferons surtout référence, dans cet article, aux auteurs consultés alors, tout en ajoutant quelques écrits plus récents. 
TABLEAU 2

\section{Devis d'évaluation}

\begin{tabular}{|c|c|c|}
\hline $\begin{array}{l}\text { Objets d'évaluation } \\
\text { du programme }\end{array}$ & Aspects principaux & $\begin{array}{l}\text { Instruments de } \\
\text { cueillette des données * }\end{array}$ \\
\hline 1. Pertinence du programme & $\begin{array}{l}\text { - caractéristiques et besoins des familles } \\
\text { - conformité entre ceux-ci et ceux identifiés } \\
\text { pour le projet }\end{array}$ & - fiche de demande de gardiennage \\
\hline 2. Degré de réalisation & $\begin{array}{l}\text { - nombre et caractéristiques des familles } \\
\text { - utilisation du service de gardiennage } \\
\text { - rencontres de groupe (nombre, contenu, } \\
\text { fonctionnement) } \\
\text { - adéquation entre besoins et programme }\end{array}$ & $\begin{array}{l}\text { - fiche de sélection des F.G. }{ }^{* *} \\
\text { - fiche de demande de gardiennage } \\
\text { - grille d'analyse des rencontres F.U. }{ }^{* * *} \\
\text { - questionnaire sur rencontres F.U. } \\
\text { - grille d'observation des rencontres F.G. } \\
\text { - questionnaire sur rencontres F.G. }\end{array}$ \\
\hline $\begin{array}{l}\text { 3. Effets sur: } \\
\text { - Parents }\end{array}$ & $\begin{array}{l}\text { - réponse du programme aux besoins ex- } \\
\text { primés } \\
\text { - degré de satisfaction } \\
\text { - influence sur réseaux sociaux }\end{array}$ & $\begin{array}{l}\text { - entrevues avec F.U. } \\
\text { - grille d'analyse rencontres F.U. } \\
\text { - questionnaire sur rencontres F.U. }\end{array}$ \\
\hline - Enfants & $\begin{array}{l}\text { - état des contacts avec milieu de vie } \\
\text { - réactions au gardiennage }\end{array}$ & $\begin{array}{l}\text { - entrevues avec F.U. } \\
\text { - entrevues avec F.G. }\end{array}$ \\
\hline — Familles-gardiennes & $\begin{array}{l}\text { - réponse du programme aux besoins } \\
\text { - degré de satisfaction } \\
\text { - influence sur réseaux sociaux }\end{array}$ & $\begin{array}{l}\text { - entrevues avec F.G. } \\
\text { - grille d'observation des rencontres F.G. } \\
\text { - questionnaire sur rencontres F.G. }\end{array}$ \\
\hline —Milieu & $\begin{array}{l}\text { - information sur le projet } \\
\text { - perception et commentaires } \\
\text { - recherche de nouveaux moyens de support }\end{array}$ & - entrevues de groupe \\
\hline
\end{tabular}

* Des renseignements détaillés sur ces divers instruments seront fournis dans le rapport final d'évaluation du projet, prévu à l'automne 1987.

** F.G. : familles-gardiennes.

*** F.U. : familles-utilisatrices. 
Nous décrirons d'abord les caractéristiques des projets-pilotes, afin de mieux situer celui auquel nous référons et d'expliquer en quoi il peut être qualifié de "pilote». Nous présenterons ensuite quelques principes des trois courants de recherche mentionnés en illustrant comment ils s'appliquent ici. Comme la recherche développementale est moins connue dans le domaine du service social, nous présenterons plus de détails sur ce courant, alors que nous ne discuterons que les grandes lignes des deux autres.

\section{"Familles gardiennes » : un projet-pilote}

Même s'il existe de nombreux projets-pilotes, nous avons trouvé peu d'ouvrages sur le sujet. Nous nous sommes alors inspirées de Suchman (1967), qui en décrit les caractéristiques et les objectifs. II suggère, entre autres, que la nature même d'une telle expérience justifie l'utilisation de plusieurs courants de recherche différents.

L'objectif primaire des projets-pilotes vise à vérifier si une action ou une intervention est souhaitable. Ils sont sources d'idées nouvelles et de programmes innovateurs; on y met l'accent sur la sélection des objectifs pertinents et réalistes, sur l'exploration et l'évaluation des divers facteurs présents, de même que sur la souplesse de l'expérimentation qui permet des modifications en cours de route (Suchman, 1967). Ils constituent en quelque sorte un compromis entre la rigueur de la recherche évaluative, qui demande un laps de temps souvent considérable alors que des besoins ou problèmes pressants exigent des éléments de réponse rapide, et les programmes nouveaux, coûteux et non évalués dont on ne connaît pas encore les bénéfices (Suchman, 1967 : 140).

Le projet auquel nous référons prend place dans un milieu à risque élevé où les interventions traditionnelles ne donnent pas toujours les résultats escomptés (McIntyre \& Lawler, 1980). Même si d'autres expériences innovatrices existent dans ce milieu (coopératives d'habitation, centre de rencontres pour personnes isolées, etc.), aucun d'entre eux ne fait l'objet d'une évaluation systématique telle qu'exigée dans un projet-pilote, c'est-à-dire avec spécification d'objectifs en termes mesurables.

En plus de faire preuve de nouveauté et de réalisme dans ses objectifs, un projet-pilote doit démontrer de façon explicite sa signification sociale (Suchman, 1967). Dans le présent cas, la signification sociale et la nouveauté se retrouvent particulièrement au niveau de l'objectif global, qui vise à dépasser la simple consommation de services pour favoriser l'établissement de nouveaux réseaux et de 
nouvelles solidarités entre les familles du milieu. Un projet-pilote doit aussi être transposable, dans le sens que certains de ses résultats devraient pouvoir s'appliquer à l'extérieur du système où il se trouve. Dans cette optique, une attention particulière est portée, dans la présente expérience, à la culture spécifique et aux valeurs du milieu dans lequel il prend place, afin d'en comprendre toute la signification et de faire ressortir les aspects qu'il faudrait ajuster avant d'implanter une intervention semblable dans un milieu différent.

\section{Liens avec la recherche développementale}

Étant donné que les projets-pilotes visent l'innovation sociale, nous nous sommes tournées vers un courant de recherche peu connu en service social, mais qui préconise cet aspect : la recherche développementale. Lors de l'étape de conception, nous nous sommes appuyées sur l'ouvrage de Rothman (1980) qui traitait de ce courant. Cet auteur a adapté au service social le modèle $R$ \& $D$ (recherche et développement) utilisé d'abord en milieu industriel pour développer de nouvelles technologies; il élabore un processus de création de réponses innovatrices aux problèmes sociaux, accompagné d'une évaluation de la démarche ainsi que des résultats préliminaires.

Plus récemment, Thomas (1985) a défini ce courant de recherche comme incluant "l les méthodes par lesquelles la technologie sociale est analysée, conceptualisée, créée et évaluée » (p. 498, traduction libre). II apporte un élément nouveau à la phase initiale du processus décrit par Rothman, et lui donne un autre nom: Developmental research and utilization (DR \& U). Même si, au début, nous nous sommes inspirées de Rothman (1980), nous considérons que la démarche telle que décrite par Thomas s'approche davantage de l'expérience que nous avons vécue.

Rothman (1980) suggère de commencer le processus de recherche développementale en étudiant les recherches empiriques déjà faites sur les problèmes sociaux afin d'en dégager des consensus et d'en appliquer les éléments théoriques à la pratique. Les diverses données retenues deviennent l'objet d'une expérimentation limitée, évaluée de façon systématique et ajustée en conséquence avant que le programme soit utilisé de façon plus étendue ou implanté dans un autre milieu (Rothman, 1980). Le processus DR \& U, par contre, reconnaît qu'il n'existe pas toujours suffisamment de recherches empiriques pour qu'on s'y réfère comme source unique de données pour guider l'innovation. Thomas (1985) indique qu'on peut se fier, entre autres, à 
l'expérience pratique que l'on a d'un problème ou d'une situation, à la politique sociale et aux lois pour trouver des solutions nouvelles.

Le projet auquel nous référons se caractérise par un accent important sur la recherche de réponses nouvelles aux problèmes du milieu. L'étude de la documentation que nous avons effectuée n'a pas permis de trouver une expérience qui en regroupe l'ensemble des éléments: un service de gardiennage dispensé par des familles du milieu, jumelé à des activités qui visent à dépasser le simple service pour développer de nouvelles solidarités dans la communauté. Il s'est alors grandement appuyé sur la connaissance que les intervenantes sociales avaient des besoins et des problèmes de la clientèle et du milieu visés, de même que sur les réflexions qui découlent de leur pratique professionnelle. À cet égard, le projet dont nous traitons se rapproche du modèle DR \& $U$.

On peut considérer le modèle développemental comme une forme particulière de recherche appliquée. Il se caractérise par des interventions souples, une ouverture aux variables émergentes et une cueillette continuelle de données (Guba, cité dans : Rothman, 1980). En même temps, il comporte des limites et des risques. Selon Mackie (cité dans : Rothman, 1980), plus une étude offre des possibilités d'applications concrètes, moins on peut en contrôler toutes les variables, ce qui constitue une limite certaine. Ceci n'implique pas, toutefois, que la recherche soit d'une qualité moindre que celles dans lesquelles on peut davantage les contrôler, à condition toutefois qu'on tienne compte des objectifs qui visent l'innovation et qu'on effectue une évaluation formative (Lewin, cité dans: Rothman, 1980).

\section{Liens avec la recherche évaluative}

On retrouve, dans les projets-pilotes, certains principes de la recherche évaluative. Dans la section qui suit, nous indiquerons l'usage que nous avons fait de ces principes, tels que soulignés par quelques auteurs, en insistant sur la recherche évaluative de type formatif.

Comme on peut le constater à partir du tableau 2, ce projet s'interroge, comme toute recherche évaluative, sur l'utilité du service et sur sa pertinence par rapport aux besoins exprimés. On veut "s'assurer que l'intervention proposée répond à un problème réel et que les solutions préconisées sont conformes aux attentes de la population" (Beaudry, 1984 : 401). Dans la présente expérience, comme c'est souvent le cas dans la pratique, ce sont des intervenantes qui ont effectué l'analyse des besoins. Il s'agit de vérifier dans quelle mesure les 
besoins invoqués par la population rejoignent ceux identifiés au début (Beaudry, 1984).

Un autre principe consiste à spécifier les objectifs de sorte que les chercheurs puissent en mesurer le degré de réalisation. À l'étape de l'élaboration du programme, on doit donc les préciser ainsi que leur séquence, en plus de clarifier les rôles de chaque membre de l'équipe (Beaudry, 1984). Dans l'élaboration du projet cité, la formulation d'objectifs clairs et mesurables et la détermination de la séquence de leur réalisation, sur les trois ans prévus pour la durée du projet, ont constitué une préoccupation majeure pour les chercheuses. Compte tenu du nombre de personnes impliquées, il a aussi fallu accorder une importance particulière à la clarification des rôles respectifs.

Cependant, les projets-pilotes diffèrent de la recherche évaluative classique sur plusieurs plans. Certains auteurs soulignent qu'il faut accorder une importance, non seulement à l'évaluation des effets, mais aussi au processus d'implantation (Beaudoin, 1982; Beaudry, 1984). Dans les projets-pilotes, on met un accent particulier sur l'appréciation du processus suivi. Dans l'expérience citée, le déroulement de l'ensemble du processus fait l'objet d'une analyse continue, afin de permettre des modifications en cours de route. Cette étude a conduit, entre autres, à des ajustements au niveau du choix et de l'utilisation de certains instruments de cueillette de données. À titre d'exemple, un des instruments prévus (Fiche personnelle de l'enfant) a été transformé après discussions avec les familles-gardiennes, lesquelles ont exprimé des craintes au sujet du respect de la confidentialité et des risques de réactions négatives des mères.

Les projets-pilotes diffèrent aussi de la recherche évaluative au niveau de leur évaluabilité. Dans ce type de recherche, il existe, selon Rutman (1982), des conditions préalables à l'évaluation des programmes : en plus de s'assurer de la précision des objectifs, il est nécessaire de posséder des renseignements détaillés sur les buts, les activités, les objectifs et les liens de causalité afin d'en dégager un consensus sur les mesures d'appréciation et sur les hypothèses avancées.

Comme les projets-pilotes sont nouveaux par définition, il est difficile d'atteindre un niveau de précision tel que celui qu'exige la recherche évaluative classique : ils s'inspirent plutôt du type formatif, qui s'appuie davantage sur une approche inductive, cherchant à établir des associations plutôt qu'à vérifier des hypothèses (Rutman, 1982). Par exemple, dans l'expérience qui nous préoccupe, certaines données préliminaires portent à penser que la répétition de contacts entre familles-gardiennes et familles-utilisatrices permet l'établissement de sentiments de confiance et une communication plus poussée entre 
elles. Ceci nous amène à insister sur l'attribution d'une même famillegardienne à l'enfant qui a déjà utilisé le service, à moins d'indications contraires. Nous devrions être davantage en mesure, à la fin du projet, d'identifier des associations et peut-être d'élaborer des hypothèses à explorer dans des recherches subséquentes.

La recherche formative utilise plusieurs moyens d'information (observations, entrevues, questionnaires) afin de clarifier et de comprendre, tout en tenant compte des contraintes du milieu (Rutman, 1982). Dans cette optique, et en raison des exigences de souplesse de la démarche, ce type de recherche insiste moins sur les notions de validité et de fiabilité, sans les négliger pour autant. On y utilise davantage des méthodes qualitatives, afin d'effectuer une analyse du processus, et en vue d'appuyer, de compléter ou de confirmer des données quantitatives (Lecomte, 1982: 143). Tel qu'indiqué au tableau 2, plusieurs instruments différents (grilles d'observation, questionnaires, entrevues, etc.) sont utilisés; des méthodes d'analyse, tant qualitative que quantitative, complètent l'évaluation.

Au niveau de l'évaluation des effets, on essaie de faire une évaluation molaire ou entière du programme afin de "brosser un portrait global de l'efficacité du programme face à ses objectifs de départ " (Beaudry, 1984 : 406). Cette démarche s'effectue, à la fois dans une perspective formative, et dans une perspective sommative.

\section{Liens avec la recherche-action}

Un contrôle limité des variables et une insistance sur la souplesse méthodologique sont caractéristiques d'un troisième courant qui influence le projet: la recherche-action, qui prend beaucoup d'ampleur depuis quelques années, et sur laquelle il existe plusieurs ouvrages récents. Au début, soit en 1984, on a retrouvé plus d'ouvrages décrivant les expériences de recherche-action que de textes en expliquant les caractéristiques et les processus. Nous nous sommes alors surtout inspirées de Stinson (1978) à cause de la clarté de son texte et de sa pertinence à notre projet. Cependant, nous ferons référence, dans la partie qui suit, à quelques ouvrages plus récents.

Malgré la diversité de méthodologies qui se regroupent sous ce terme, on peut en dégager certains points communs. En plus des éléments déjà mentionnés dans le paragraphe précédent, on y retrouve une distanciation plus ou moins grande par rapport au modèle expérimental, une situation dans le contexte naturel, l'implication des sujets dans la recherche et une conception du temps en spirale plutôt que linéaire (Charbonneau, 1985 ; Goyette et Lessard, 1985). 
Ces derniers constatent, dans leur recension des écrits, que les auteurs nord-américains insistent plus que les auteurs européens sur le développement et l'état des programmes. Certains mettent l'accent sur le processus d'apprentissage de l'ensemble des personnes qui y sont impliquées et sur le développement communautaire qui accompagne la recherche-action (Goyette et Lessard, 1985).

Dans ce projet-pilote, le processus d'apprentissage mutuel revêt une importance particulière. Les chercheurs expliquent aux responsables le langage de la recherche afin qu'ils soient en mesure d'en utiliser les résultats et de participer aux décisions; ils tentent aussi de les aider à systématiser leur action et à développer leur compétence, entre autres dans l'organisation du travail, l'animation des rencontres de groupe, ainsi que l'utilisation de leurs ressources et de celles du milieu. De son côté, le collectif transmet aux chercheurs sa connaissance approfondie des caractéristiques du milieu, de ses valeurs, de ses normes et de ses besoins; il contribue également à faciliter le déroulement des opérations d'évaluation en informant les familles-utilisatrices, avec lesquelles il a une relation privilégiée, et en les invitant à collaborer. Par la vigilance dont il fait preuve, il aide l'équipe de recherche à mettre en pratique les grands principes théoriques de la participation communautaire et du respect de l'autonomie des personnes et des groupes.

Plusieurs auteurs constatent que la recherche-action est une réaction à la sous-utilisation de la recherche traditionnelle par les groupes communautaires, laquelle s'explique par la mystification qui l'entoure et parce que les chercheurs en ont souvent davantage profité que les sujets eux-mêmes (Stinson, 1978; Goyette et Lessard, 1985). La recherche-action se caractérise par la responsabilité du chercheur de rendre des comptes au groupe (accountability), par son engagement dans le projet, par sa reconnaissance d'une influence mutuelle de la recherche et de l'action, et par un équilibre entre l'accent mis sur le résultat et celui accordé au processus (Stinson, 1978; Dubost, 1983; Charbonneau, 1985 ; Goyette et Lessard, 1985).

Dans le présent projet, une collaboration étroite s'est développée graduellement entre les divers partenaires, tant au niveau de l'action que de l'évaluation. Par exemple, dès le début, les chercheurs ont accordé beaucoup d'importance à la vulgarisation du processus de recherche auprès des familles du milieu, à leur participation à la formulation des objectifs et à la préparation des instruments de cueillette des données. Les résultats sommaires de l'évaluation sont également discutés avec les familles afin d'ajuster le déroulement du projet en conséquence. Ces dernières discutent régulièrement avec les chercheurs du volet action, des difficultés qu'elles rencontrent, des 
questions qu'elles se posent, des résultats qu'elles obtiennent, des initiatives qu'elles envisagent. Chacune des parties met à contribution ses propres compétences. Dans ce type de projet, le chercheur ne vise pas à être "objectif" mais bien à s'impliquer dans une relation symbiotique avec le milieu.

\section{Quelques résultats préliminaires}

Comme le suggèrent les courants de recherche sur lesquels s'appuie le projet, différentes données sont analysées tout au long de son déroulement. Ainsi, la fiche de demande de gardiennage a fait l'objet d'une première compilation. Les données recueillies jusqu'à maintenant, quoique partielles, permettent de croire que le service de gardiennage rejoint la clientèle visée. Les familles utilisatrices correspondent, en effet, au portrait-type d'une famille "à risque»: parmi les 110 familles qui avaient déjà utilisé le service au moment de l'analyse sommaire, $80 \%$ étaient monoparentales, sous la responsabilité d'une femme âgée de moins de 35 ans et qui a un ou deux enfants. Plus de la moitié des enfants gardés était d'âge pré-scolaire. Quatre-vingt douze pour cent des familles vivaient de prestations gouvernementales.

La fiche de sélection des familles-gardiennes a été révisée avec le collectif, à la lumière de leur expérience et des apprentissages faits après approximativement un an et demi de fonctionnement; cette révision a permis de systématiser davantage les critères de sélection et de les pondérer, en plus de modifier la procédure d'évaluation des familles : la sélection des nouvelles familles-gardiennes et la réévaluation annuelle de celles déjà engagées dans le projet se fait maintenant par deux membres du collectif qui remplissent la fiche séparément, à partir d'observations précises, et en discutent ensuite, avant de prendre une décision.

Les données recueillies lors des entrevues effectuées avec les familles utilisatrices ont été aussi compilées sommairement. On peut considérer que, dans l'ensemble, les mères identifient, comme effets du service de gardiennage, un repos physique et psychologique; certaines mentionnent aussi une diminution de l'anxiété reliée à l'isolement, suite à cette forme de sécurité que procure le service. Elles disent également apprécier la rapidité avec laquelle elles peuvent obtenir le service et l'absence de procédures compliquées.

Un questionnaire rempli par vingt-cinq familles utilisatrices lors d'une rencontre de groupe a permis de dégager certaines attentes par rapport à de telles rencontres. Les résultats sont mis à la disposition du 
Collectif et devraient aider à la préparation d'un programme de rencontres adapté aux besoins et intérêts des familles.

L'analyse de la grille d'observation et du questionnaire aux familles-gardiennes fournit également des éléments de réflexion et des pistes de travail afin d'améliorer le fonctionnement des rencontres dans le sens des objectifs visés.

En somme, la cueillette des données et leur analyse même sommaire, tout au cours du déroulement du projet, fournissent des éléments de nature à consolider l'action, à l'ajuster ou à la réorienter, selon les besoins.

\section{Conclusion}

Le fait de s'appuyer sur plusieurs courants de recherche contribue à élargir la perspective d'ensemble du projet et lui permet de tenir compte des multiples facettes qu'il recouvre tout en l'adaptant le plus possible au contexte spécifique dans lequel il se déroule. De la recherche évaluative formative, on retient, entre autres : l'importance à accorder autant au processus qu'aux résultats, la nécessité de vérifier l'utilité du service et sa pertinence par rapport aux besoins exprimés, de même que la recherche de liens d'association entre éléments du processus et résultats. La recherche développementale, surtout celle de type DR \& $U$, nous invite à chercher des réponses innovatrices aux problèmes d'un milieu en nous appuyant, non seulement sur des études formelles, mais sur des observations et des réflexions des gens concernés. Ce modèle invite aussi à une grande souplesse méthodologique et à une cueillette continue de données, laquelle favorise un ajustement de l'action au fur et à mesure de son déroulement. Cette souplesse méthodologique se retrouve aussi dans le modèle de recherche-action qui insiste, en plus, sur l'importance de l'apprentissage mutuel des diverses parties engagées dans le projet. L'image qu'on peut en dégager est celle d'un vaste chantier dans lequel chacun fait profiter l'autre de ses connaissances et compétences et se développe à travers cet échange mutuel.

L'utilisation de divers courants de recherche risque, par contre, d'entraîner certaines difficultés; mentionnons, entre autres, celle de conserver une unité et une cohérence suffisante dans les objectifs de divers ordres à atteindre et dans les aspects multiples de la démarche retenue. L'établissement de priorités dans les objectifs et la démarche, qui rejoignent les valeurs et les intérêts des diverses parties impliquées, constitue un autre défi de taille lorsqu'on veut à la fois atteindre des 
résultats șignificatifs et à long terme, en même temps que respecter les besoins et l'autonomie de chacun. Cela signifie concrètement qu'il faut accepter que les résultats ne soient ni instantanés ni spectaculaires mais que chacun ait le temps d'expérimenter de nouvelles attitudes et de nouveaux comportements, de les évaluer et de les discuter avant d'en arriver à des changements durables.

Tout cela exige, de part et d'autre, patience, ouverture d'esprit, disponibilité, dialogue, respect du rythme et de l'autonomie de chacun.

\section{Références bibliographiques}

BEAUDOIN, A., "La réalisation de l'évaluation dans la pratique : critères et limites ", dans: R. LeCOMTE et L. RUTMAN (éds), Introduction aux méthodes de recherche évaluative, Québec, Les Presses de l'Université Laval, 1982: 155-176.

BEAUDRY, J., "L'évaluation de programme", dans: B. GaUthier (éd.), Recherche sociale. De la problématique à la collecte des données, Sillery, Les Presses de l'Université du Québec, 1984 : 389-415.

Bellamy, D. et M. ShOOKNER, "Developing and implementing a policy of prevention in children's services in Ontario ". Helping Networks and the Welfare State, Toronto, University of Toronto, 1980:22-53. ("Case Studies and Supplementary Materials».)

Charbonneau, R., "Vers une définition de la recherche-action", dans: J.P. DESLAURIERS (éd.), La recherche qualitative : résurgence et convergences, Chicoutimi, Université du Québec à Chicoutimi, Groupe de recherche et d'intervention régionales, 1985 : 109-122. ("Renouveau méthodologique».)

Darveau-Fournier, L., A. Home et C. Bédard, "La collaboration avec le milieu : outil de formation et de recherche", Revue canadienne de service social, 1986 : 145-156.

DuBOSt, J., "Les critères de la recherche-action ", Pour, 90, 1983 : 17-21.

GARBARINo, J. et D. SHERMAN, "High-risk neighborhoods and high-risk families: The human ecology of child maltreatment ", Child Development, vol. 51, no 1, $1980: 188-198$.

GottueB, B.H. (éd.), Social Networks and Social Support, Beverly Hills, Sage Publications, 1981.

GoyetTe, G. et M. LeSSARD, La recherche-action : ses fonctions, ses fondements et son instrumentation, Québec, Conseil québécois de la recherche sociale, 1985.

Jenkins, S. et M. Sauber, Paths to Child Placement: Family Situations Prior to Foster Care, New York, Department of Welfare, The Community Council of Greater New York, 1966.

LeCOMTE, R., "Les apports de l'évaluation qualitative et critique en recherche évaluative", dans: R. LeCOMTE et L. RUTMAN (éds), Introduction aux 
méthodes de recherche évaluative, Québec, Les Presses de l'Université Laval. 1982 : 143-153.

MCINTYRE, E. et P. LAWLER, "Preliminary study of child care networks in a high risk locality", Helping Networks and the Welfare State, Toronto, University of Toronto, 1980 : 302-318. ("Symposium Papers".)

Rothman, J., Social $R \&$ D : Research and Development in the Human Services, Englewood Cliffs, (N.J.), Prentice-Hall, 1980.

RUtMAN, L., "La recherche formative et l'évaluabilité de programme", dans: R. LECOMTE et L. RUTMAN (éds), Introduction aux méthodes de recherche évaluative, Québec, Les Presses de l'Université Laval, 1982 : 65-77.

Stinson, A., Action Research for Social Action, Ottawa, Centre for Social Welfare Studies, Carleton University, 1978. ("Occasional Paper ", 2/78).

SuCHMAN, E., Evaluative Research, New York, Russell-Sage, 1967.

Thomas, E.J., "Developmental research», dans : R. Grinnell (éd.), Social Work Research and Evaluation, Itasca (III.), Peacock, 1985 : 483-499. 